Sexes of infants born after induction of ovulation

\begin{tabular}{lrr}
\hline Source* & Girls & Boys \\
\hline F J Beernink (private communication) & 4 & 12 \\
J B Brown (private communication) & 104 & 104 \\
S Byrne (private communication) & 40 & 41 \\
R N Turksoy et al & 2 & 5 \\
R S Neuwirth et al & 1 & 3 \\
G C Liggins and H K Ibertson & 1 & 4 \\
R A Aiken & 2 & 4 \\
M C Botha et al & 4 & 0 \\
R D Atlay and G W Pennington & 2 & 1 \\
N Eckstein and M P David & 4 & 2 \\
W J Garrett et al & 5 & 4 \\
W Mobius and W Carol & 4 & 4 \\
M Granat & 4 & 0 \\
Giovannucci Uzielli et al & 4 & 2 \\
L Varga et al al Ouist & 2 & 3 \\
J Cohen and C Quist & 0 & 2 \\
J C Monnier et al & 1 & 4 \\
T A Walker and W F Baden & 2 & 0 \\
\hline Total & 186 & 195 \\
\hline
\end{tabular}

*Full references are available on request.

come known to me since that publication. There is no evidence for heterogeneity among these various sets of data so it seems reasonable to pool them. This yields 522 boys and 601 girls. When tested against an expected sex ratio of 514 , this sample gives a chi-squared value of $10.86, p \bumpeq 0.001$. So taken together the data still powerfully support the hypothesis.

As the techniques of hormonal monitoring improve, the amount of administered hormone will presumably decrease. So if the hypothesis is correct, the departures from expected sex ratios among children born following induced ovulation should become progressively less. Working with mice, Elbling ${ }^{3}$ has given strong evidence that under some circumstances the administration of gonadotrophin is associated with a subsequent low sex ratio in the pups. Smith and Chrisman 5 failed to reproduce this result; but such is the strength of Elbling's evidence that one might infer not that the hypothesis may be safely dismissed but that a phenomenon does exist which is incompletely understood.

It would be useful if other workers would repeat Elbling's experiment. I should also be glad to be contacted by anyone with unpublished data on the sex of infants following induced ovulation.

I am grateful to Dr F J Beernink (of Berkeley, California), Professor J B Brown (University of Melbourne), and Sister S Byrne (of the Women's Hospital, Surrey Hills, New South Wales) for data in tabulated form.

WILLIAM H JAMES

University College London

MRC Mammalian Development Unit,
London NW $12 \mathrm{HE}$

1 James WH. Br Med $\mathcal{F}$ 1980;281:711-2.

2 James WH. Lancet 1980;1:1124-6. Elbling L. Proc Soc Exp Biol Med 1975;149:376-9.
smith CM, Chrisman CL. Nature 1975;253:631-2.

\section{Reduction of free testosterone by}

\section{antiepileptic drugs}

SIR,-This tidy research work by Dr Jila Dana-Haeri and others (9 January, p 85) prompted me to contemplate the role of sex hormones in epileptics with acne vulgaris. Typical adolescent acne is found in epileptic boys under treatment long before puberty, and at one time I even thought that phenytoin had a virilising effect as epileptic women taking it had hirsutism. Adult epileptics are prone to very severe acne, which is resistant to local applications that are usually helpful; and this might imply a more severe sex hormone imbalance in epileptics.
The endocrine factors causing acne are complex, but androgens seem to play a dominant role. Acne is not seen in the castrated, and antiandrogens produce improvement albeit with loss of libido. Furthermore oral contraceptives, provided that they contain a non-androgenic progestogen, afford beneficial results in both sexes. It would seem therefore that acne results from an imbalance of sex hormones with a relative preponderance of androgens; and epileptics under treatment tend to suffer from early, severe, and stubborn acne.

Bearing in mind these clinical observations, I remain somewhat bewildered by the findings of Dr Dana-Haeri and others, who found a reduced concentration of plasma free testosterone in epileptics under treatment with antiepileptic drugs.

IAN MARTIN-SCOTT

Chorleywood, Herts WD3 4BW

\section{Squash ball to eye ball}

SIR,-May I add to the correspondence concerning squash ball eye injuries with a comment on injury to the eye from a squash racket?

Fourteen years ago I was hit by a squash racket in the left eye, which resulted in severe loss of vision due to a retinal haemorrhage. Not wishing to give up playing squash I had a metal mask constructed (figure). It does not

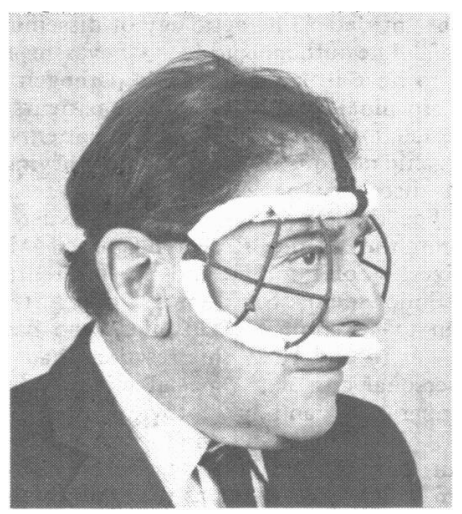

Protective mask for a squash player.

obstruct the vision in any way and will prevent both a squash ball and a squash racket from damaging the eye. If anyone would be interested in further information I would be happy to supply it.

E D KERR

Barn Cottage,

Cryers Hill Lane,

Widmer End

High Wycombe, Bucks

\section{Smoking, IgE, and occupational allergy}

SIR,-Dr O Zetterström and his colleagues (7 November, $p$ 1215) report that raised serum IgE concentrations and increased risk of occupational allergy may represent another hazard of smokers. Our recent work (reported to the 15th Italian National Congress of Allergology and Clinical Immunology, 1981) seems in part to confirm this finding and to provide additional stimulus for further research on this new subject.

In a survey carried out in collaboration with Professor G Berlinguer (Institute of Physiology and
Occupational Hygiene, University of Rome) to assess damage induced by an occupational exposure to cosmetics, serum IgE levels were measured in 320 hairdressers and 201 controls by PRIST (Phadebas IgE, Pharmacia, Uppsala). Lung function tests were also carried out. IgE concentration in the serum was related to the age of the subjects, the presence of current or previous allergic symptoms, occupational exposure to cosmetics, and smoking habits (subjects smoking more than five cigarettes a day being considered as smokers) Statistical analysis was performed on the natural logarithm (In) of the IgE values by Professor L Piccinato (Institute of Probability, Faculty of Statistics, University of Rome) by regression analysis.

While total serum IgE was not influenced by the age of the subjects or exposure to cosmetics, a history of allergy and, more interestingly, smoking habits were significantly related to high $\operatorname{IgE}$ values $(p<0.05)$. No significant additive effect was observed between the two variables. Mean levels of serum IgE in smokers and non-smokers are shown in the table.

Serum IgE levels in smokers and non-smokers

\begin{tabular}{lcc}
\hline & No & In IgE (mean \pm SD) \\
\hline Smokers & 222 & $4 \cdot 12 \pm 1 \cdot 57$ \\
Non-smokers & 283 & $3 \cdot 79 \pm 1.33$ \\
< 0.05. & & \\
\hline
\end{tabular}

Professor Zetterström and his colleagues report that raised $\operatorname{IgE}$ values are due to an increased frequency of sensitisation in smokers, possibly related to the damage inflicted by smoking on the bronchial mucosa. This was not manifest in our data, the frequency of allergy being in fact similar in smokers and non-smokers. On the contrary, the statistical analysis we performed showed that the effect of smoking on IgE levels was independent of the occurrence of clinical sensitisation. Moreover, even when allergic subjects were excluded from the sample, the 133 smokers still had higher IgE concentrations than the 182 non-smokers $(4.09$ 土 $1.56 v 3.69+1.28 \ln \mathrm{IgE} ; \mathrm{p}<0.05)$. This aspect also emerged from the study of $\mathrm{Dr}$ Zetterström and his co-workers, the difference in IgE serum levels between smokers and non-smokers reaching an even higher statistical significance when only non-atopic subjects were considered.

Smoking habits may certainly influence the target organ of a clinical hypersensitivity (that is, the bronchi), thus possibly explaining the higher frequency of allergic asthma in smokers. ${ }^{1}$ However, on the basis of our findings, we would like to suggest the possibility of a more direct effect of smoking on IgE production, apart from that of an increased frequency of sensitisation to smoke or occupational or common allergens. Daniele and coworkers reported alterations in smokers in the morphology and function of alveolar macrophages and a reduced proliferative lymphocyte response to phytohaemagglutinin and concanavallin A. ${ }^{2}$ This could imply a toxic effect of smoking on various immune cells, including those involved in IgE synthesis. Smoking has in fact been shown to cause significant changes in other immunoglobulin classes. $^{3}$

It is commonly reported that the distribution of serum IgE values in healthy subjects is not Gaussian. In our opinion this implies an inadequacy of the criteria used for selecting normal subjects, as yet unrecognised environmental or pathological factors being responsible for the heterogeneity of the so-called "normal" population. The effect of smoking on IgE suggests that smokers should be excluded from all studies of normal IgE levels. Our study of a large number of subjects provides prompt confirmation of the reports of Gerrard and coworkers ${ }^{4}$ and of Professor Zetterström and his colleagues that healthy subjects should bear in mind the risk that smoking, among other things, could increase their IgE levels. The 
lack of an effect of smoking on IgE in allergic subjects should not be a licence for them to smoke.

SERGIO BONINI

University of Rome Medical Clinic I,

Umberto Polyclinic I,

00161 Rome

Kiviloog J, Irnell L, Eklund G. Scand $\mathcal{F}$ Respir Dis $1974 ; 55: 262-76$

Daniele RP, Dauber JH, Altose MD, Rowlands DT
Jr, Gorenbrg DJ. Am Rev Respir Dis 1977;116 997-1002.

3 Gulsvik A, Fagerhol MK. Lancet 1979 ;i :449.

Gerrard JW, Heneir DC, Ko CG, Mink J, Meyers A,

\section{Mucosal injury and recurrent aphthous stomatitis}

SIR,-I wish to express condemnation of the experiment by Dr David Wray and others (12 December, p 1569). Thirty patients with recurrent aphthous ulceration and 15 "healthy controls" had their buccal mucosa deliberately damaged either by anaesthetic injected into the long buccal nerve, by insertion of sutures which were left for 24 hours, or by piercing with towel tenaculum. The procedures produced two puncture wounds.

Was it honestly explained to all the patients that marked pain and discomfort was likely and that the exercise was purely academic and not for any possible immediate benefit to the sufferers ? I know from experience that careful questioning of many patients with aphthous ulceration will elicit the information that any minor trauma-for example, from certain foods-may produce ulceration. There is no need to prove the point in such a cruel manner.

There is no successful treatment of this condition and the experimental physician must never assume that he is the acknowledged agent of the interests of future sufferers from that condition. This appears to me yet another case of choosing martyrs for possible good that may result.

M H PAPPWORTH

London NW3

**We sent this letter to the authors, and Dr Wray replies below.-ED, $B M Y$.

SIR,-Dr Papworth's comments are pertinent, not only to research into recurrent aphthous stomatitis but also to broader questions regarding the whole of human experimentation. One's defence for such methods is, however, easily justified.

With particular reference to the subjects examined in our studies, the National Institutes of Health, where the research was conducted, were conceived specifically for the purpose of furthering medical science and have no service commitment to their patients, who therefore attend for the purpose of participating in clinical research only. Scrutinisation of research protocols, including explicit consent forms, is carried out by peer review groups prior to the initiation of clinical studies. All patients were therefore fully aware of the implications of their involvement in this trial. Our thanks to these volunteers should be emphasised for their selfless participation.

Clinicians, like Dr Pappworth, have long suspected that trauma plays a role in the initiation of recurrent aphthous stomatitis. In contradistinction to his comments, however, it is unfortunately no longer acceptable to base clinical judgment on the hearsay of patients, and a well-controlled study on the role of trauma was indeed indicated not only to aid in clarifying the aetiology of recurrent aphthous stomatitis but also to warn dental practitioners of the problems associated with executing even the simplest manipulations in these patients. Some successes have been achieved in the management of recurrent aphthous stomatitis, although the treatment remains unsatisfactory.

While we wait for the frontiers of medicine to be conquered, we cannot shun the onus of providing heritable information with each investigation or treatment of a patient. In this respect all patients are martyrs for a better tomorrow.

Glasgow G63 90P

D WRAY

\section{Masturbation and fornication}

SIR,-Dr J O Drife gives an interesting summary of the pros and cons of masturbation as against fornication (2 January, p 39). In real life, however, most people indulge in both. Masturbation, which occurs in at least $90 \%$ of men and $30 \%$ of women by the age of $20,{ }^{1}$ acts as a learning process that acquaints the individual with sexual response and orgasm. Girls do not rely so heavily as boys on fantasy and usually do not experience such a voluptuous response. ${ }^{2}$ Responsible fornication-that is, intercourse between willing couples who take sensible precautions -adds an extra dimension to one's sexual experience because it involves another person's response. This can be just as important in the learning process as masturbation. Whereas many couples do remain virgins until marriage, and have happy and fulfilled sexual lives, this by no means applies to everyone. Not a few patients appear at psychosexual clinics, having "waited until marriage" only to find themselves trapped with a totally incompatible partner. Montaigne reminds us of the difficulties engendered when women "buy a cat in a poke" and he goes on to excuse their infidelities for this reason. ${ }^{3}$ Certainly "irresponsible" fornication is littered with hazards: but so is irresponsible anything, from drunken driving to overeating, yet we do not condemn eating or drinking out of hand.

I would disagree with Dr Drife's view about "little fresh scientific evidence appearing in the last 40 years." Kinsey's massive studies alone involved 18000 subjects, ${ }^{14}$ while the research of Masters and Johnson has helped to elucidate the physiological response of sexua expression $^{56}$; and there have been studies too numerous to list since. The general conclusion suggest that physiologically masturbation in both sexes goes through the same pathways as coitus to orgasm, although there is often a different psychological response to the two. Freud's theory ${ }^{7}$ about the existence of separate clitoral and vaginal orgasms in women, and the "immaturity" of the former, has been shown not to be true, and indeed multiple orgasm have been described in many women. Dr Drife's analogy of irresponsible intercourse and masturbation to cigarette smoking and pipe smoking made me smile; but perhaps the analogy for "responsible" fornication compared with masturbation should be going to Covent Garden with an attractive companion to see a full-blooded, live performance compared with watching an opera alone on television-you may get mugged or run over on the way, but most people are prepared to take that slight risk.

Manchester M22 4BR

R E GoodmaN

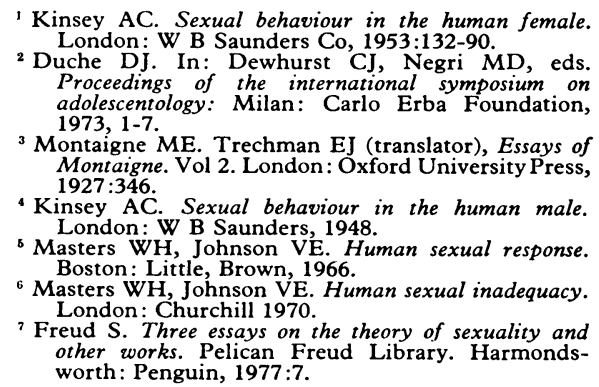

\section{Simple technique for measuring serum or plasma viscosity with disposable} apparatus

SIR,-I am grateful to Dr Jonathan Kay (28 November, $p$ 1471) for his criticisms of my paper (31 October, p 1154). I have the opportunity now to publish the correlation data which had been submitted with the original article.

Dr Kay requests information on the withinindividual correlation as a method of comparing the two techniques for measuring viscosity. It is somewhat unsatisfactory to correlate the within-individual values at each separate temperature as the numbers available and the range of values are small. In addition, there is the problem of relying on the Ostwald apparatus as a standard due to the intrinsic inaccuracy of timing. However, the numbers of data points and the range of values can be extended by aggregating the results from the three temperatures provided that the data from an individual serum sample are included only once (that is, at only one temperature). These correlations are illustrated in the accompanying table, where it will be noted that there are no

Comparison of Ostwald with disposable apparatus in measuring relative serum viscosity (within individual correlations)

\begin{tabular}{cc}
\hline Temperature & $\begin{array}{c}\text { Linear correlation } \\
\text { coefficient }\end{array}$ \\
\hline $4^{\circ} \mathrm{C}(\mathrm{n}=9)$ & $0 \cdot 76$ \\
$21^{\circ} \mathrm{C}(\mathrm{n}=14)$ & $0 \cdot 71$ \\
$37^{\circ} \mathrm{C}(\mathrm{n}=30)$ & $0 \cdot 70$ \\
$37^{\circ}+21^{\circ} \mathrm{C}(\mathrm{n}=30)$ & 0.84 \\
$4^{\circ} \mathrm{C}(\mathrm{n}=25)$ & 0.93
\end{tabular}

samples measured at $4^{\circ} \mathrm{C}$ that had not been measured at $21^{\circ} \mathrm{C}$. A second, minor point: $\mathrm{Dr}$ Kay mentions my publishing mean values when, of course, the scattergraphs clearly show median values, no assumptions having been made about a normal or even a symmetrical distribution.

The disposable apparatus, widely available (and cheap), should probably be further evaluated as a design suited to measurement of fluid viscosity. In clinical studies it has already proved useful in the assessment of myeloma serum viscosity and further studies can easily be carried out in other diseases characterised by abnormal plasma or serum viscosity.

ROBERT LEONARD

Sidney Farber Cancer Institute,

Boston 